\title{
Partitions with Distinct Multiplicities of Parts: On An "Unsolved Problem" Posed By Herbert Wilf
}

\author{
James Allen Fill* \\ Department of Applied Mathematics and Statistics \\ The Johns Hopkins University \\ 3400 N. Charles Street \\ Baltimore, MD, USA 21218-2682 \\ jimfill@jhu.edu \\ http://www.ams.jhu.edu/ fill/ \\ Svante Janson \\ Department of Mathematics \\ Uppsala University \\ P. O. Box 480 \\ SE-751 06 Uppsala, Sweden \\ svante.janson@math.uu.se \\ http://www. math.uu.se/ svante/ \\ Mark Daniel Ward ${ }^{\dagger}$ \\ Department of Statistics \\ Purdue University \\ 250 N. University Street \\ West Lafayette, IN, USA 47907-2066 \\ mdw@purdue .edu \\ http://www. stat.purdue.edu/ mdw/
}

Submitted: Mar 13, 2012; Accepted: May 16, 2012; Published: May 21, 2012

Mathematics Subject Classifications: 05A16, 05A17, 68W40

\begin{abstract}
Wilf's Sixth Unsolved Problem asks for any interesting properties of the set of partitions of integers for which the (nonzero) multiplicities of the parts are all different. We refer to these as Wilf partitions. Using $f(n)$ to denote the number of Wilf partitions, we establish lead-order asymptotics for $\ln f(n)$.
\end{abstract}

Keywords: asymptotic enumeration, partitions of integers, Wilf partitions

Dedicated to the memory of Herbert S. Wilf (1931-2012).

*J. A. Fill's research was supported by the Acheson J. Duncan Fund for the Advancement of Research in Statistics.

${ }^{\dagger}$ M. D. Ward's research was supported by NSF Science \& Technology Center grant CCF-0939370. 


\section{The Problem}

Herbert S. Wilf was an expert in many areas of combinatorics. Besides writing numerous papers and books, he was a friend and mentor to many colleagues. Herb often asked intriguing questions that opened up whole new areas of investigation. In the later years of his life, he posted a set of eight Unsolved Problems on his webpage Wilf (2010). At the time of Herb's death in January 2012, only one of these problems was solved (the third problem: see Ward (2010)). In this paper, we discuss Wilf's sixth "Unsolved Problem."

\section{Distinct multiplicities Wilf (2010)}

Let $T(n)$ be the set of partitions of $n$ for which the (nonzero) multiplicities of its parts are all different, and write $f(n)=|T(n)|$. See Sloane's sequence A098859 for a table of values. Find any interesting theorems about $f(n)$. The mapping that sends a partition of $n$ to another partition of $n$ in which the roles of parts and multiplicities are interchanged is a well defined involution on $T(n)$, which is how I arrived at the study of this problem.

\section{Definitions}

We refer to partitions in which the (nonzero) multiplicities of the parts are all different as Wilf partitions.

Define

$$
\mathcal{M}_{r}:=\left\{\left(m_{1}, m_{2}, \ldots, m_{r}\right): m_{k} \text { are distinct positive integers }\right\}
$$

and

$$
\mathcal{P}_{r}:=\left\{\left(p_{1}, p_{2}, \ldots, p_{r}\right): p_{k} \text { are distinct positive integers with } p_{1}<\cdots<p_{r}\right\} .
$$

Then the set of Wilf partitions of $n$ is

$$
T(n):=\bigcup_{r \geqslant 1}\left\{(\mathbf{m}, \mathbf{p}): \mathbf{m}=\left(m_{1}, \ldots, m_{r}\right) ; \mathbf{p}=\left(p_{1}, \ldots, p_{r}\right) ; m_{1} p_{1}+\cdots+m_{r} p_{r}=n\right\} .
$$

We write $p(n, r)$ for the number of partitions of $n$ into $r$ parts, and $d(\cdot)$ to denote the divisor function, i.e., $d(n)$ is the number of divisors of $n$.

Wilf defines $f(n)=|T(n)|$ and then asks to find anything interesting about $T(n)$.

\section{Main Result}

Theorem 3.1. Let $f(n)$ denote the number of partitions of $n$ with distinct (nonzero) multiplicities. Then

$$
\ln f(n) \sim \frac{6^{1 / 3}}{3} n^{1 / 3} \ln n \sim(6 n)^{1 / 3} \ln \left[(6 n)^{1 / 3}\right] \quad \text { as } n \rightarrow \infty .
$$




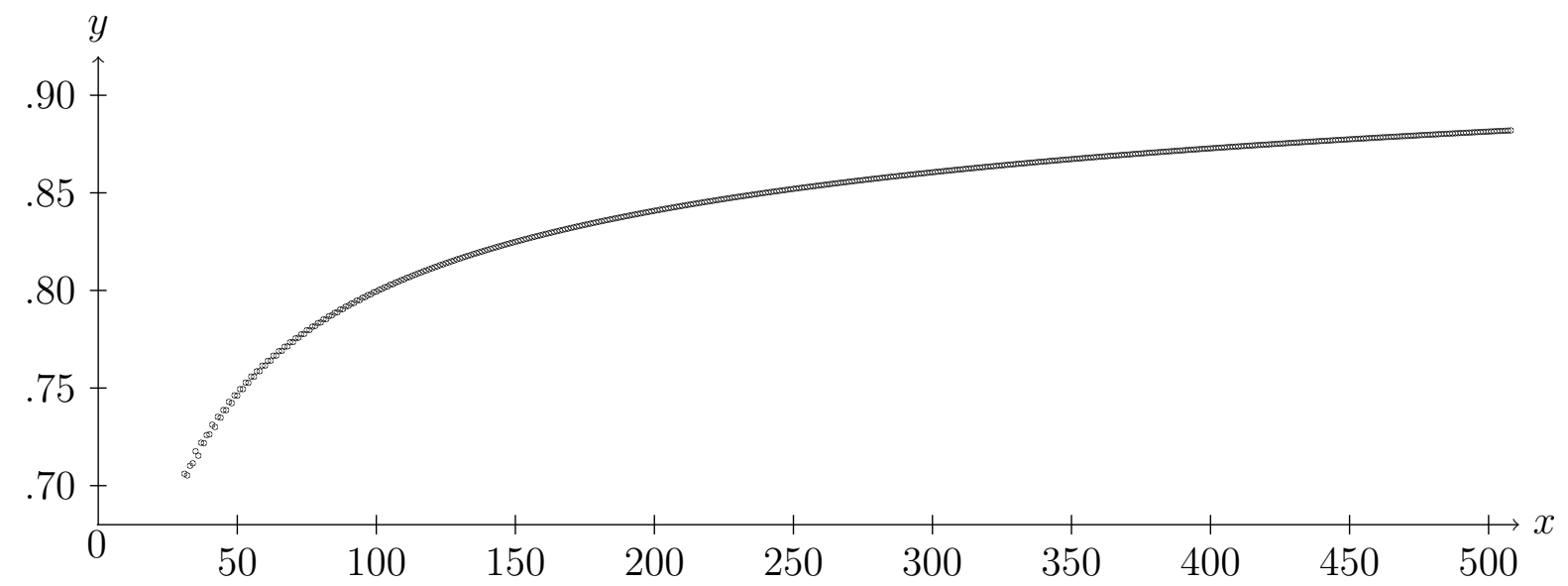

Figure 1: Plot of the values of $\frac{\ln f(n)}{(6 n)^{1 / 3} \ln \left[(6 n)^{1 / 3}\right]}$ for $31 \leqslant n \leqslant 508$.

The theorem will be established by matching upper and lower bounds for $\ln f(n)$ in Lemmas 4.2 and 4.3 below.

In Figure 1, we plot the values of

$$
\frac{\ln f(n)}{(6 n)^{1 / 3} \ln \left[(6 n)^{1 / 3}\right]}
$$

for $31 \leqslant n \leqslant 508$.

\section{Proofs}

Lemma 4.1. The number $r$ of distinct multiplicities in a Wilf partition of $n$ is at most $(6 n)^{1 / 3}$.

Proof. For a given positive integer $r$, the smallest possible $n$ admitting a Wilf partition with $r$ distinct multiplicities is obtained by taking:

- multiplicity $m_{1}=r$ for part $p_{1}=1$;

- multiplicity $m_{2}=r-1$ for part $p_{2}=2$;

- multiplicity $m_{3}=r-2$ for part $p_{3}=3$;

- $\quad \vdots$

- multiplicity $m_{r}=1$ for part $p_{r}=r$. 
This yields

$$
n=\sum_{i=1}^{r}(r+1-i) i=\frac{1}{6} r^{3}+\frac{1}{2} r^{2}+\frac{1}{3} r .
$$

Hence $r \leqslant(6 n)^{1 / 3}$. This completes the proof of Lemma 4.1 .

Lemma 4.2. An upper bound for $\ln f(n)$ is

$$
\ln f(n) \leqslant(1+o(1)) \frac{6^{1 / 3}}{3} n^{1 / 3} \ln n .
$$

Proof. For each Wilf partition of $n$, put the terms in decreasing order according to the values of the products $m_{i} p_{i}$. If two terms are equal, break the tie by writing in decreasing order of the multiplicities. This gives a canonical way to write the Wilf partitions. For instance,

$$
27=8+3+3+2+2+2+1+1+1+1+1+1+1
$$

can be written as

$$
27=(1 \times 8)+(7 \times 1)+(3 \times 2)+(2 \times 3)=m_{1} p_{1}+m_{2} p_{2}+m_{3} p_{3}+m_{4} p_{4} .
$$

Notice that the products are, respectively $8,7,6,6$. The ordering of the two terms each with product 6 was decided by writing those terms in decreasing order of the multiplicities.

With this representation in mind, it follows that the number $f(n, r)$ of Wilf partitions of $n$ with $r$ distinct multiplicities is no larger than

$$
p(n, r) \times[\max \{d(j): 1 \leqslant j \leqslant n\}]^{r} .
$$

Severin Wigert (1907) [see also (Hardy and Wright, 1960, Th. 317, Chap. XVIII.1)] showed that

$$
\limsup _{n \rightarrow \infty} \frac{\ln d(n)}{(\ln n) /(\ln \ln n)}=\ln 2 .
$$

Therefore, there exists a constant $C$ such that, provided $n$ is sufficiently large, $f(n, r)$ is no larger than

$$
p(n, r) \times \max _{3 \leqslant j \leqslant n}\left\{\exp \left(r C \frac{\ln j}{\ln \ln j}\right)\right\}=p(n, r) \times \exp \left(r C \frac{\ln n}{\ln \ln n}\right) .
$$

Since by Lemma 4.1 we have $r \leqslant(6 n)^{1 / 3}$ if $f(n, r)>0$, the second factor here does not contribute to the lead-order logarithmic asymptotics for $f(n)$. Now we utilize Exercise 7.2.1.4-34 in Knuth (2005), which concerns $p(n, r)$ and is stated (in our notation) for $r \leqslant n^{1 / 3}$; but Knuth's argument is easily checked to hold also for $r \leqslant(c n)^{1 / 3}$ for any constant $c$. Choosing $c=6$ we find, for all $r \leqslant(6 n)^{1 / 3}$,

$$
p(n, r)=O\left(\frac{n^{r-1}}{r !(r-1) !}\right) \leqslant \exp \left[(1+o(1))(1 / 3)(6 n)^{1 / 3} \ln n\right]
$$

as $n \rightarrow \infty$, and then (1) yields the same estimate for $f(n, r)$. The proof of Lemma 4.2 is completed by a summation over $r \leqslant(6 n)^{1 / 3}$. 
Lemma 4.3. A lower bound for $\ln f(n)$ is

$$
\ln f(n) \geqslant(1+o(1)) \frac{6^{1 / 3}}{3} n^{1 / 3} \ln n .
$$

Proof. Let $a<6^{1 / 3}$, and let $K$ be a fixed large integer. Let $b:=\left\lfloor a n^{1 / 3} / K\right\rfloor$ and divide the interval $[1, K b] \subseteq\left[1, a n^{1 / 3}\right]$ into $K$ equal parts $I_{1}, \ldots, I_{K}$. Consider only permutations $\left(p_{1}, \ldots, p_{K b}\right)$ of $[1, K b]$ that map $I_{j}$ into $I_{K+1-j}$ for every $j \in\{1, \ldots, K\}$. For such permutations, if

$$
a=[6(1-2 \epsilon)]^{1 / 3},
$$

and if $K$ is large enough (depending on $\epsilon$ but not on $n$ ), then $\sum_{i} i p_{i}<(1-\epsilon) n$, and we obtain (if $n$ is large enough) a Wilf partition by taking $i$ parts of size $p_{i}$ for each $2 \leqslant i \leqslant K b$ and a single part of size $n-\sum_{i=2}^{K b} i p_{i}$.

Thus the number of Wilf partitions of $n$ is at least

$$
b !^{K}=\exp [K b(\ln b+O(1))]=\exp \left[a n^{1 / 3}\left(\ln n^{1 / 3}+O(1)\right)\right]=\exp \left[\left(\frac{a}{3}+o(1)\right) n^{1 / 3} \ln n\right] .
$$

This completes the proof of Lemma 4.3 , since we may take $a$ arbitrarily close to $6^{1 / 3}$.

\section{Open Problems}

We have found the first-order asymptotic description of $\ln f(n)$, but lower-order terms of $\ln f(n)$ remain unknown. A Herculean task would be to find the first-order asymptotic description of $f(n)$ itself. As a much simpler task, David S. Newman has mentioned that it would be nice to have a proof that $f(n)$ is nondecreasing.

\subsection{An involution}

Wilf (2010) mentions a mapping on $T(n)$ in which the roles of parts and multiplicities are interchanged. We let $\sigma_{n}$ denote this mapping. Hence, $\sigma_{n}((\mathbf{m}, \mathbf{p}))$ is

$$
\left(\mathbf{p}_{\pi}, \mathbf{m}_{\pi}\right)=\left(\left(p_{\pi(1)}, \ldots, p_{\pi(r)}\right),\left(m_{\pi(1)}, \ldots, m_{\pi(r)}\right)\right),
$$

where $\pi$ is the permutation making $m_{\pi(1)}<\cdots<m_{\pi(r)}$. For instance, two partitions of 83 are

$83=1+1+1+1+1+1+1+4+4+4+4+5+5+5+5+5+5+5+5+5+5+5+5$

and

$$
83=4+4+4+4+7+12+12+12+12+12 .
$$

More succinctly, we can write these partitions as elements of $T(83)$ as $((7,4,12),(1,4,5))$ and $((4,1,5),(4,7,12))$. Then we have

$$
\sigma_{83}:((7,4,12),(1,4,5)) \mapsto((4,1,5),(4,7,12)),
$$


and, reversing the roles of the multiplicities and partitions, we have

$$
\sigma_{83}:((4,1,5),(4,7,12)) \mapsto((7,4,12),(1,4,5)) .
$$

Switching the roles of the parts and multiplicities again, we get back to the original partition, so $\sigma_{n}$ is seen to be an involution. Note that $\sigma_{n}$ has order 2 on most elements of $T(n)$, but $\sigma_{n}$ has order 1 on some elements. In particular, $\sigma_{n}$ fixes every $(\mathbf{m}, \mathbf{p})$ for which $m_{i}=p_{i}$ for all $i$, for example,

$$
\sigma_{65}:((2,5,6),(2,5,6)) \mapsto((2,5,6),(2,5,6)) ;
$$

but there are also other examples, such as

$$
\sigma_{10}:((1,2,3),(3,2,1)) \mapsto((1,2,3),(3,2,1)) .
$$

It is an open problem to find (asymptotics for) the number of fixed points of $\sigma_{n}$.

\section{Acknowledgements}

Daniel Kane has independently obtained similar results. We thank David S. Newman and Vladeta Jovovic for early conversations about efficient ways to generate the numbers in Wilf's sequence. We also thank David S. Newman for early feedback on the manuscript. The sequence can be accessed in Neil Sloane's database, The Online Encyclopedia of Integer Sequences [Sloane (2008)]. Maciej I. Wilczyński has recently generated the first 500 values in Wilf's sequence. The current list of known values of Wilf's sequence is found at http://oeis.org/A098859/b098859.txt.

\section{References}

G. H. Hardy and E. M. Wright. An Introduction to the Theory of Numbers. 4th ed., Oxford Univ. Press, Oxford, 1960.

D. E. Knuth. The Art of Computer Programming, Volume 4A: Combinatorial Algorithms, Part 1. Addison-Wesley Professional, 2011.

N. J. A. Sloane. The On-Line Encyclopedia of Integer Sequences, 2008. Sequence A098859: https://oeis.org/A098859.

M. D. Ward. Asymptotic rational approximation to Pi: Solution of an "unsolved problem" posed by Herbert Wilf. Discrete Mathematics and Theoretical Computer Science, AM (2010), 591-602.

S. Wigert. Sur l'ordre de grandeur du nombre des diviseurs d'un entier. Arkiv Mat. Astr. Fys. 3 (1907), paper 18, 1-9.

H. Wilf. Some unsolved problems, December 13, 2010. Available for download as a pdf file, http://www.math.upenn.edu/ wilf/website/UnsolvedProblems.pdf. 